\title{
Multiple Arbitrary Shape Via-Hole and Air-Bridge Transitions in Multilayered Structures
}

\author{
Ming-Ju Trai, Member, IEEE, Chinglung Chen, Member, IEEE, \\ Nicolaos G. Alexopoulos, Fellow, IEEE, and Tzyy-Sheng Horng, Member, IEEE
}

\begin{abstract}
A methodology for the design of multiple via-hole and air-bridge transitions of arbitrary shape in multilayered multiport microstrip circuits is presented in this paper. Application of multiple via holes to the design of microstrip filters and other devices will be discussed. To properly describe the current along the vertical post, the simple pulse function with a triangular cross section is used in the moment method analysis. Circularly and rectangularly shaped vertical transitions are analyzed for several practical applications. Comparisons of numerical results with experimental and available analytical data show good agreement.
\end{abstract}

\section{INTRODUCTION}

$\mathbf{C}$ OMPACT SIZE and large scale integration of electronic devices have been driving the trend toward a multilayered interconnection system. Via holes and other vertical shunt posts, such as bond wires and air bridges, are increasingly important in microwave integrated circuit/monolithic-microwave integrated circuit (MIC/MMIC) design. Via holes are used to connect parallel microstrip lines for signal transmission between different levels. Vias can be modeled by lumped circuit elements at lower frequencies. The equivalent circuits of vias based on the quasi-static analysis have been investigated by Wang et al. [1], [2]. At higher frequencies the propagation characteristics of via holes have a stronger electromagnetic effect on the performance of devices, therefore, rigorous analysis is necessary to predict frequency response correctly. Full-wave analysis and modeling has been carried out by the finite-difference time-domain (FDTD) approach [3], [4], the transmission-line matrix method [5], the mode-matching technique [6], the spectral-domain analysis (SDA) [7], [8], and the matrix-penciled moment method [9]. Most of these analyses are limited to rectangular discretization or the thinwire approximation. In this paper, a combined mixed-potential integral equation (MPIE) and electric-field integral equation (EFIE) technique is presented to model vias of arbitrary shape. The MPIE method [10]-[12] combined with the triangular patch expansion function [13] has been proven efficient to model arbitrarily shaped planar geometries [14], [15]. To model the vertical current along the vias, a simple pulse

Manuscript received March 28, 1996. This research was supported in part by U.S. Army Research Grant DAAH 04-93-G-0228 and Hughes-UC Microelectronics Contract 94-005.

M.-J. Tsai is with Lucent Technologies, Murray Hill, NJ 07974 USA.

C. Chen is with the Anritsu/Wiltron Company, San Jose, CA 95123 USA.

N. G. Alexopoulos is with the Electrical Engineering Department, University of California, Los Angeles, CA 90095 USA.

T.-S. Horng is with the Electrical Engineering Department, National Sun Yat-Sen University, Kaohsiung, 80424 Taiwan, R.O.C.

Publisher Item Identifier S 0018-9480(96)08555-9.

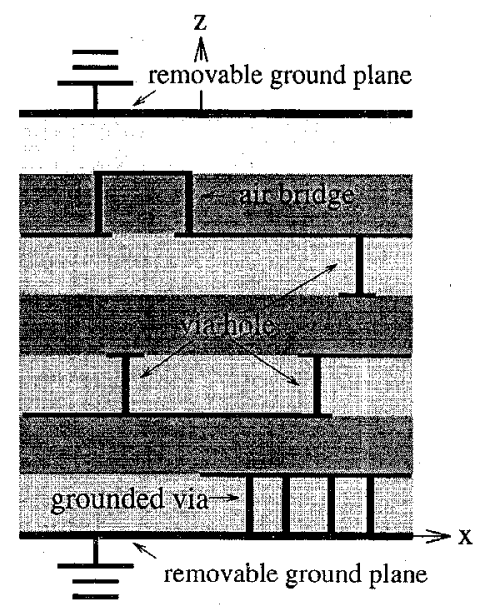

Fig. 1. Generic via-hole and air-bridge transitions in a multilayered medium.

function with a triangular cross section is used to approximate the current density. The EFIE formulation is adopted to evaluate the interaction between vias since the integration over the vertical axis can be done analytically. The main advantage of using EFIE here is that only one less-singular Green's function $\left(G_{E}^{z z^{\prime}}\right)$ is involved, so it simplifies the formulation and computation.

The combined MPIE-EFIE method presented in this paper meshes the whole microstrip geometry with small triangular facets. The MPIE formulation is used to evaluate the selfcoupling terms of planar subdomains as well as the mutualcoupling terms between planar and vertical cells. The selfcoupling submatrix due to the vertical posts is calculated from the EFIE formulation. These theoretical analyses will be detailed in Section II. Several examples with via-hole applications are analyzed and discussed in Section III.

\section{COMBINED MPIE-EFIE FORMULATION}

A generalized three-dimensional (3-D) multilayered microstrip circuit is shown in Fig. 1. The medium is assumed to be infinite in the $x-y$ plane, and microstrip patterns are assumed to be of infinitesimal conductor thickness. Both the upper and lower ground planes are removable to represent either a shielded, semi-open, or open structure. Multiple vias as well as air bridges are used to connect different microstrips. Grounded vias are also applied to achieve the short effect.

The construction of an EFIE is the major step toward solving a pertinent problem. An EFIE can be set up by applying the 
boundary condition of zero tangential field on metal surface $S$ as

$$
\begin{aligned}
0 & =\hat{n} \times\left[\vec{E}^{\text {inc }}(\vec{r})+\vec{E}^{s}\left(\vec{r} \mid \vec{r}_{s}\right)\right] \\
& =\hat{n} \times\left[\vec{E}^{\text {inc }}(\vec{r})+\int_{s} \overline{\bar{G}}_{E}\left(\vec{r} \mid \vec{r}_{s}\right) \cdot \vec{J}_{s}\left(\vec{r}_{s}\right) d S_{s}\right]
\end{aligned}
$$

where $\vec{r}$ is on microstrips. $\overline{\bar{G}}_{E}$ is the dyadic electric-field Green's function, and $\vec{J}_{s}$ is the surface current distribution. For simplicity, we neglect conductor and dielectric losses. If the magnetic vector potential $(\vec{A})$ and charge scalar potential $(V)$ as $\vec{E}=-j \omega \vec{A}-\nabla V$ are introduced, then (1) can be rewritten as an MPIE

$$
\begin{aligned}
\hat{n} \times \vec{E}^{\text {inc }}(\vec{r})= & \hat{n} \times[j \omega \overline{\bar{A}}(\vec{r})+\nabla V(\vec{r})] \\
= & \hat{n} \times\left[j \omega \int_{s} \overline{\bar{G}}_{A}\left(\vec{r} \mid \vec{r}_{s}\right) \cdot \vec{J}_{s}\left(\vec{r}_{s}\right) d S_{s}\right. \\
& \left.+\nabla \int_{s} G_{q}\left(\vec{r} \mid \vec{r}_{s}\right) q_{s}\left(\vec{r}_{s}\right) d S_{s}\right]
\end{aligned}
$$

where $\overline{\bar{G}}_{A}$ and $G_{q}$ are the dyadic Green's functions for $\vec{A}$ and $V$, respectively. First, both Green's functions are derived for multilayered structures in the spectral domain analytically, then evaluated in the spatial domain using Sommerfeld integrals [16]. $\vec{J}_{s}$ and $q_{s}$ are the unknown distributions of electric surface current and charge. The reason for using MPIE instead of EFIE is that the spatial-domain Green's functions $\overline{\bar{G}}_{A}$ and $G_{q}$ are less singular than $\overline{\bar{G}}_{E}$, so the computations become simpler and more stable. However, the integration over the vertical basis function can alleviate the singular behavior of $\overline{\bar{G}}_{E}$, therefore, the MPIE (2) is used to model the mutual coupling between planar elements, while the EFIE (1) is used for vertical mutual-coupling effects.

The method of moments is applied to convert the integral equations to an algebraic linear system. The first step is to expand the unknown current distribution with a set of basis functions. To model the planar current of arbitrarily shaped microstrip geometries, we adopt the triangular patch subdomain function [13], shown in Fig. 2(a), and defined as

$$
\overrightarrow{t r i}_{n}(\vec{r})= \begin{cases}\frac{\ell_{n}}{2 A_{n}^{+}}\left(\vec{r}-\vec{r}_{n 1}\right)=\frac{\ell_{n}}{2 A_{n}^{+}} \vec{\rho}_{n}^{+} ; & \vec{r} \in T_{n}^{+} \\ \frac{\ell_{n}}{2 A_{n}^{-}}\left(\vec{r}_{n 4}-\vec{r}\right)=\frac{\ell_{n}}{2 A_{n}^{-}} \vec{\rho}_{n}^{-} ; & \vec{r} \in T_{n}^{-} \\ 0 ; & \text { otherwise }\end{cases}
$$

where $T_{n}^{ \pm}$denote the domains of two adjacent triangles with area $A_{n}^{ \pm}$. The $n_{\text {th }}$ expansion function is uniquely associated with a pair of triangles sharing a common edge (interior edge) whose length is $\ell_{n}$. This basis function can describe vector current flow, and it automatically satisfies the boundary conditions on microstrips because only tangential components exist along the boundary edges. Its divergence is constant over the associated triangular face which is equivalent to a constant-charge basis function.

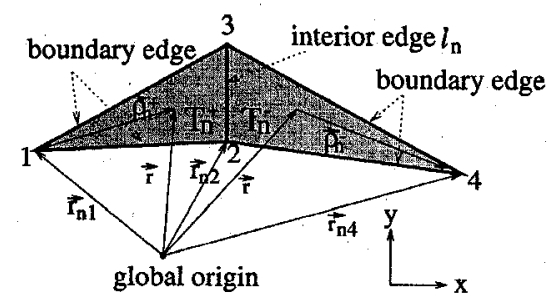

(a)

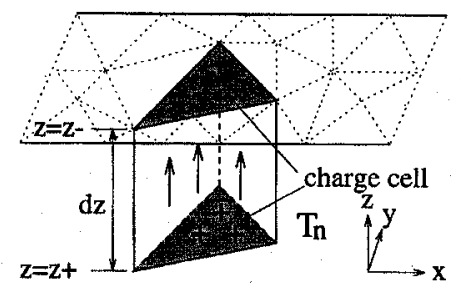

(b)

Fig. 2. Current expansion functions. (a) Triangular patch function $(\overrightarrow{t r i})$ for planar current; (b) vertical volume current function $(\overrightarrow{v e r})$ for current along vias.

To model the vertical current along the vias, a vertical volume current function with a triangular cross section, shown in Fig. 2(b), is used in this analysis. It is defined as

$$
\overrightarrow{v e r}{ }_{n}(\vec{r})= \begin{cases}\hat{z} \frac{1}{d_{z}} ; & \vec{r} \in T_{n} \\ 0 ; & \text { otherwise }\end{cases}
$$

where $d_{z}$ is the height of the vertical basis function in the $\hat{z}$ direction. Its divergence shows two associated charge cells uniformly distributed on the top and bottom faces, as shown in Fig. 2(b). Any vertical posts with either a rectangularly, circularly, or arbitrarily shaped cross section can be expanded by using this proposed basis function. This basis function is modified from the similar function with a rectangular cross section [7]. It is noted that the $1 / d_{z}$ term is used in the definition of the vertical currents because of the consistency of length-unit-dimension in the impedance matrix elements. In addition, this volume basis function is valid for the via size which is small compared to the wavelength. That is, the surface current on the small via is approximated by the uniformly distributed volume current.

The planar current $\left(\vec{I}_{H}\right)$ on the planar microstrips, and the vertical current $\left(\vec{I}_{V}\right)$ along the vias can be expanded as

$$
\vec{I}_{H}=\sum_{n=1}^{N_{H}} A_{n} \overrightarrow{t r i} i_{n}, \quad \vec{I}_{V}=\sum_{n=1}^{N_{V}} B_{n} \overrightarrow{v e r} r_{n}
$$

respectively. $N_{H}$ and $N_{V}$ are the unknown numbers of the planar and vertical cells. $A_{n}$ and $B_{n}$ are unknown expansion coefficients. After testing with the same functions (Galerkin's procedure), a system of linear equations can be obtained as

$$
\begin{gathered}
{\left[\begin{array}{ll}
{\left[Z_{H H}\right]_{N_{H} * N_{H}}} & {\left[Z_{H V}\right]_{N_{H} * N_{V}}} \\
{\left[Z_{V H}\right]_{N_{V} * N_{H}}} & {\left[Z_{V V}\right]_{N_{V} * N_{V}}}
\end{array}\right]_{T * T}\left[\begin{array}{l}
{[A]_{N_{H}}} \\
{[B]_{N_{V}}}
\end{array}\right]_{T}} \\
\quad=\left[\begin{array}{c}
{[V]_{N_{H}}} \\
{[0]_{N_{V}}}
\end{array}\right]_{T}
\end{gathered}
$$

where $T=N_{H}+N_{V}$ is the total number of unknowns. $\left[Z_{H H}\right],\left[Z_{H V}\right],\left[Z_{V H}\right]$, and $\left[Z_{V V}\right]$ are self- and mutual- 
coupling submatrices between two different basis functions. $\left[Z_{H H}\right]$ based on MPIE (2) can be expressed as

$$
\begin{aligned}
Z_{H H}^{m n}= & j \omega \int_{S_{m}} d S \int_{S_{n}} d S_{s}\left[\overrightarrow{\operatorname{tri}} i_{m}(\vec{r}) \cdot \overline{\overline{G_{A}}}\left(\vec{r} \mid \vec{r}_{s}\right)\right. \\
& \left.\cdot \overrightarrow{\operatorname{tri}}_{n}\left(\vec{r}_{s}\right)\right]+\frac{1}{j \omega} \int_{S_{m}} d S \int_{S_{n}} d S_{s}\left[\nabla \overrightarrow{\operatorname{tr}} i_{m}(\vec{r})\right] \\
& \cdot G_{q}\left(\vec{r} \mid \vec{r}_{s}\right)\left[\nabla_{s} \cdot \overrightarrow{t r i}_{n}\left(\vec{r}_{s}\right)\right] .
\end{aligned}
$$

The Green's function for the vector potential $\overline{\bar{G}}_{A}$ is coupled with planar current basis function $\overrightarrow{t r i}$, while $G_{q}$ is coupled with the charge distribution $\left(\nabla_{s} \cdot \overrightarrow{t r i}\right)$.

The self-coupling term $\left[Z_{V V}\right]$ can be expressed from the EFIE in the following form:

$$
\begin{aligned}
Z_{V V}^{m n} & =\int_{S_{m}} \int_{S_{n}}\left[\int_{z} \int_{z_{s}} \overrightarrow{v e r_{m}} \cdot \overline{\overline{G_{E}}} \cdot \overrightarrow{v e r_{n}} d z d z_{s}\right] d S d S_{s} \\
& =\frac{1}{d_{z} d_{z s}} \int_{S_{m}} \int_{S_{n}}\left[G_{E}^{z z^{\prime}}\right] d S d S_{s}
\end{aligned}
$$

where $G_{E}^{z z^{\prime}}$ is the Green's function analytic $\hat{z} \hat{z}$ component for the electric field after performing the integration over $z$ and $z_{s}$. The original Green's function $G_{E}^{z z}$ has the more singular near-field behavior as $O\left(1 / \rho^{2}\right)$. However, since the simple pulse function is used for $\overrightarrow{v e r}$, the integration over the vertical axis can be done analytically. As a result, the new integrand $G_{E}^{z z^{\prime}}$ is less singular as $\bar{G}_{A}$ and $G_{q}$ to $O(1 / \rho)$, so it is easy to evaluate $G_{E}^{z z^{\prime}}$ through the Sommerfeld integral. Therefore, no charge distribution or scalar potential is involved in these terms. The formulation of $G_{E}^{z z^{\prime}}$ for single-layered media is expressed in the Appendix.

The mutual coupling terms $\left[Z_{H V}\right]$ and $\left[Z_{V H}\right]$ are transposes of each other. $\left[Z_{V H}\right]$ can be written as

$$
\begin{array}{r}
Z_{V H}^{m n}=\int_{S_{m}} \int_{S_{n}}\left[\int_{z} \overrightarrow{v e r} r_{m} \cdot \overline{\overline{G_{E}}} \cdot \overrightarrow{\operatorname{tri} i_{n}} d z\right] d S d S_{s} \\
=\frac{1}{d_{z}} \int_{S_{m}} \int_{S_{n}}\left\{\left[j \omega G_{A}^{v h^{\prime}}+\frac{G_{q}\left(z^{+}\right)-G_{q}\left(z^{-}\right)}{j \omega}\right]\right. \\
\left.\cdot\left(\nabla \overrightarrow{\operatorname{tr} i_{n}}\right)\right\} d S d S_{s}
\end{array}
$$

where the Green's functions $G_{A}$ and $G_{q}$ of vector and scalar potentials from MPIE are used. Instead of using $G_{A}^{z x}$, we introduce $G_{A}^{v h}$ with the relationships $G_{A}^{z x}=\partial G_{A}^{v h} / \partial x$ and $G_{A}^{z y}=\partial G_{A}^{v h} / \partial y$. Similarly $G_{A}^{v h^{\prime}}$ denotes the analytic expression of $G_{A}^{v h}$ after the integration over the vertical length of testing basis $\overrightarrow{v e r}_{m}$. The interaction between current basis functions can be eliminated by using the vector identity, so only charge distribution $\left(\nabla_{s} \overrightarrow{t r} i_{n}\right)$ is involved in (9). They are listed in the Appendix for a simple single-layered structure.

In this analysis, the excitation mechanism is a series deltagap voltage source attached at the end of the input line. Therefore, only one nonzero element exists in the excitation vector [V], and it is normalized to be $1 \mathrm{~V}$. Once the matrix elements and excitation vector are determined, the current distribution can be solved, and all the circuit parameters can be obtained.
The most time-consuming steps of this algorithm are evaluating the matrix elements and solving the matrix equation. In this analysis, the standard subroutine DLINCG for a general complex linear equation is utilized from the IMSL Mathematical Library at the University of California at Los Angeles (UCLA) Office of Academic Computing (OAC) Center. To accelerate the process of filling the matrix, several numerical techniques are applied [17]-[19]. First, the Green's functions are evaluated and stored as numerical tables versus the radial distance for interpolation to accelerate the computation of reaction integrals. All the matrix elements are divided into nonsingular and singular parts. The singular part comes from the singularity of the spatial-domain Green's function for field points that are very close to the source point. It can be evaluated by four seven-point quadratures combined with the analytic expressions. The nonsingular part is approximated by a three-point average. The process of filling the impedance matrix is based on faces instead of interior edges to eliminate many redundant calculations. For example, it takes only $39.2 \mathrm{~s}$ of central-processing unit (CPU) time to solve a 555-unknown problem on an IBM RS/6000 workstation. The step of matrix inversion takes about half of the CPU time, while the evaluation of matrix elements takes the rest in this case. As the unknowns increase, the matrix solve-time becomes the dominant factor in the computation.

\section{NUMERICAL RESUltS AND Discussions}

Several examples with via-hole/air-bridge applications are discussed in this section. All computations are performed on the cluster system of IBM RS/6000's in the UCLA OAC Center.

\section{A. Grounded Via in an Infinite Microstrip Line}

The first example is an infinite microstrip line grounded by a via, which was presented in [20] by using the planar waveguide model. The structure and analyzed results are shown in Fig. 3. Three different simulations are investigated: 1) rectangular ground via expanded by one vertical current basis with a rectangular cross section; 2) rectangular ground via expanded by two vertical current bases with a triangular cross section; and 3) circular ground via expanded by eight vertical current bases with a triangular cross section. The reference plane is along the center of the ground via. The reflection coefficient of an infinite microstrip line without ground via should be close to zero. However, after adding the ground via, which is expanded by the new developed basis function, the reflection coefficient now has a magnitude close to one and a phase close to $180^{\circ}$. This means that most of the signal is reflected back by the ground via. All cases show that the current flows down to the ground plane, and a good short can be achieved over a broad frequency range.

\section{B. Spiral Inductor with an Air Bridge}

The spiral inductor is analyzed and compared to the spectraldomain analysis and measurement [7] for verification of our 


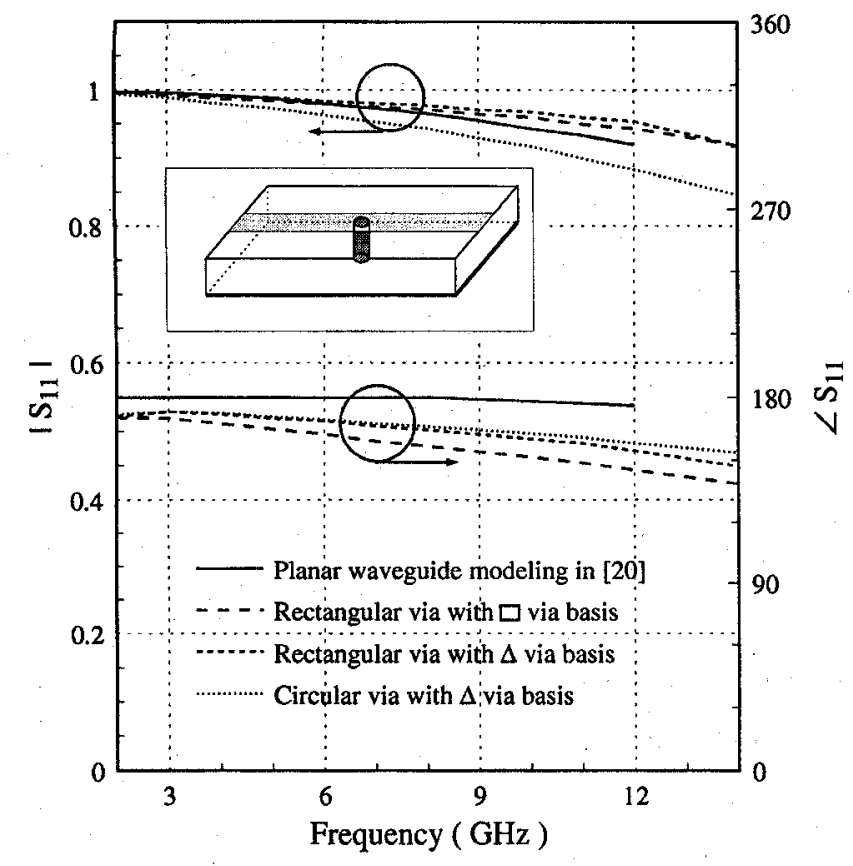

Fig. 3. Magnitude and phase of $S_{11}$ for an infinite microstrip line with a ground via. $\epsilon_{r}=10.0$, thickness $=0.635 \mathrm{~mm}$, line width $=3.0 \mathrm{~mm}$, and via-hole diameter $=1.22 \mathrm{~mm}$.

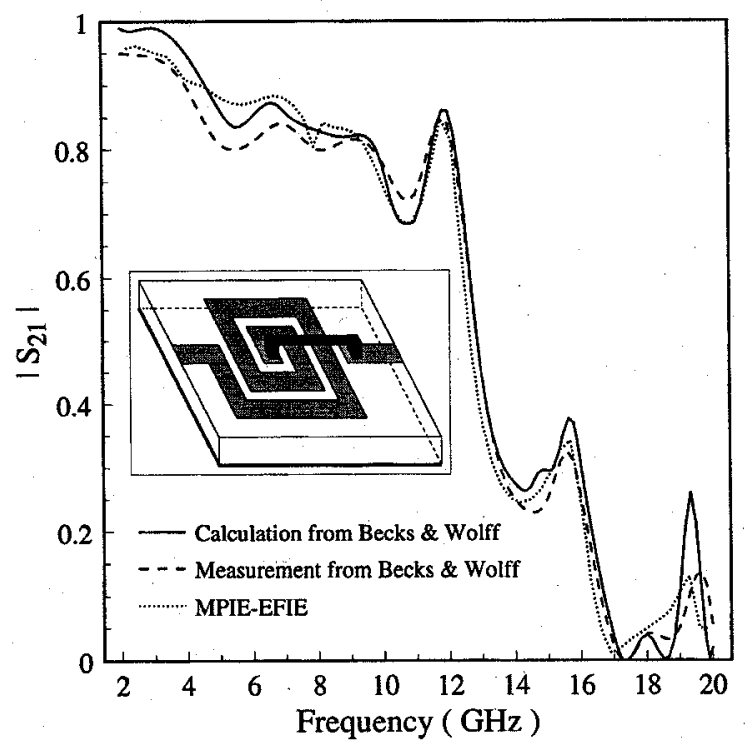

Fig. 4. Transmission coefficient of spiral inductor with an air bridge. $\epsilon_{r}=9.8$, thickness $=0.635 \mathrm{~mm}$, line width $=0.625 \mathrm{~mm}$, gap width $=0.3125 \mathrm{~mm}$, air-bridge height $=0.3175 \mathrm{~mm}$, and air-bridge width $=$ $0.3125 \mathrm{~mm}$.

method. The rectangular air bridge is composed of two triangular cells. The lengths of the microstrip lines in the input/output ports are set to be $24 \mathrm{~mm}$ to extract the scattering parameters from the dominant-mode propagation region. This circuit is discretized into 992 triangular facets, which is equivalent to 1313 unknown interior edges. The CPU time is approximately 4.5 min per frequency point. Figs. 4-6 demonstrate the excellent agreement in the scattering parameters between this analysis and the measurement.

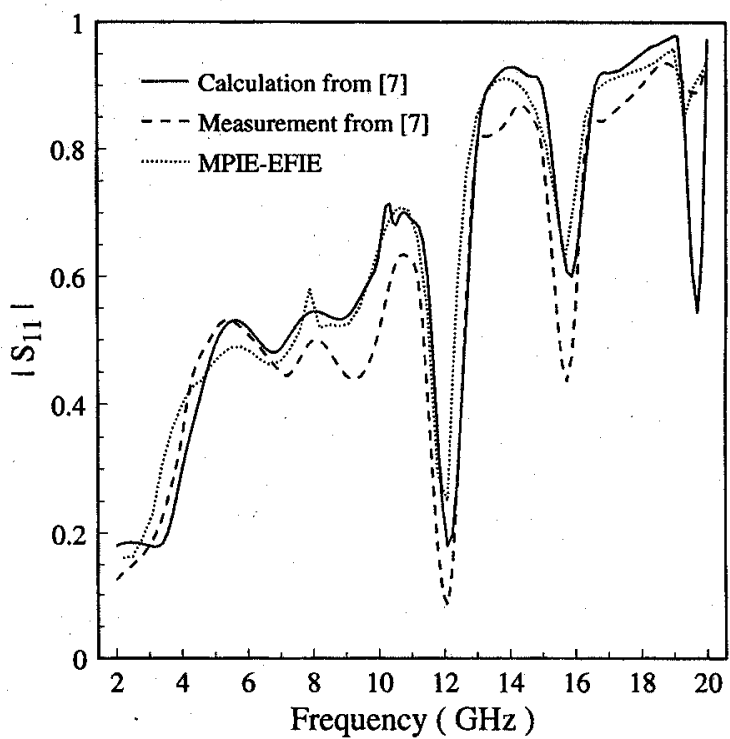

Fig. 5. Reflection coefficient (from port 1) of spiral inductor with an air bridge.

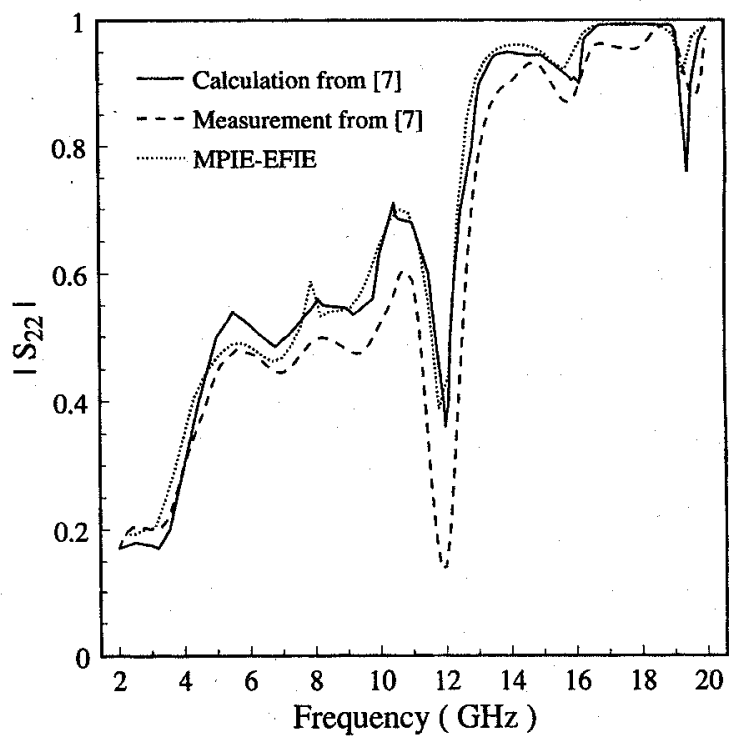

Fig. 6. Reflection coefficient (from port 2) of spiral inductor with an air bridge.

\section{Vias in Filter Design}

Multiple via holes can be applied to microstrip filter design. As an example, the bandpass filter configuration (shown in [21, Fig. 7]) is considered. Two mitered-bend lines with rectangular pads are connected to the main transmission line. Each pad is grounded by a circular via hole with a $100-\mu \mathrm{m}$ diameter. A triangular mesh is also plotted in Fig. 7. Eighteen triangular cells are used to expand the vertical current for each via hole. The total unknowns are 555, and the CPU time is about $39.2 \mathrm{~s}$ per frequency point. Compared to $444 \mathrm{~s} /$ freq for the Microwave Explorer 1.11 on HP730 [21], our algorithm is much more efficient. The simulated results are shown for lossless layers, perfectly conducting microstrip lines in Fig 8. The resonant frequency is predicted very well at $13.5 \mathrm{GHz}$ [21]. 


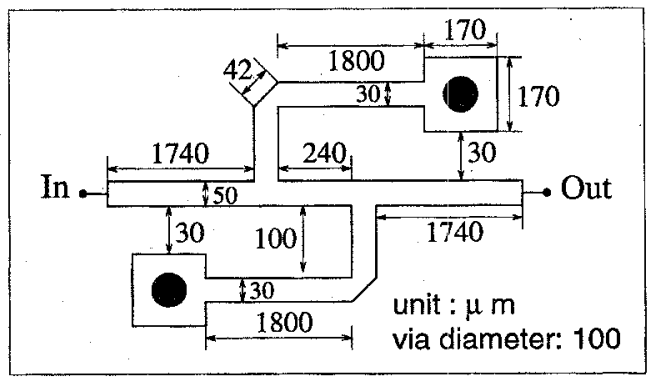

Fig. 7. Configuration and discretization of bandpass filter with two via-hole grounds (substrate height $=125 \mu \mathrm{m}$ and dielectric constant $=12.9$ ).

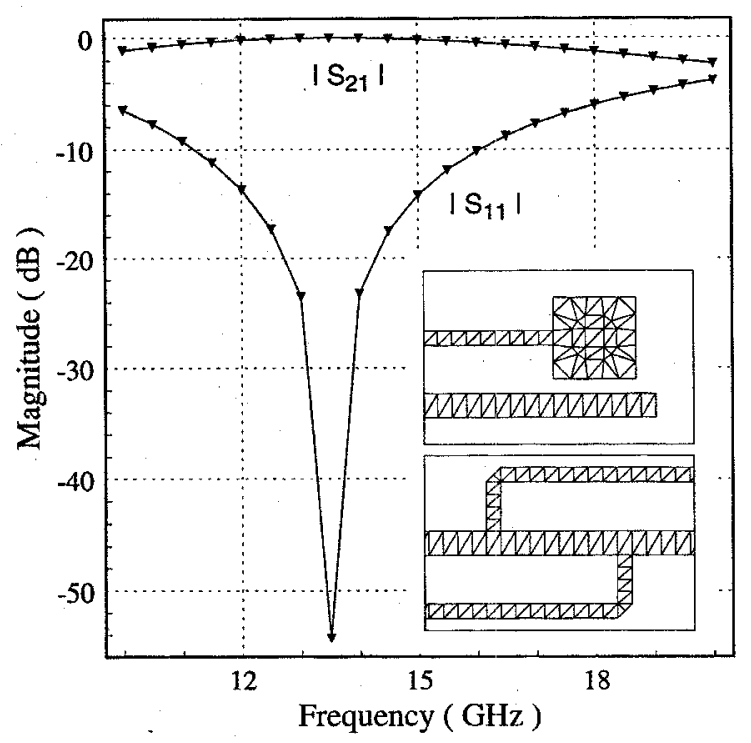

Fig. 8. Reflection and transmission coefficients of bandpass filter with two via-hole grounds.

\section{Grounded Coplanar Waveguide Circuit}

The next two examples are grounded coplanar waveguide (GCPW) test circuits proposed, fabricated, and tested by Hughes Aircraft Company. These circuits are among those that Hughes uses to interconnect various MMIC chips in multichip modules (MCM's) such as microwave transmit/receive (T/R) modules. The first example is a back-to-back, abrupt GCPW to microstrip transition circuit pair shown in Fig. 9. 50- $\Omega$ lineimpedance circuits were fabricated on 1 in by 0.5 in by 25 -mil thick alumina substrates with a thick-film process. 5-mil gaps separate the 10-mil wide center conductor from the ground return strips. The CPW ground return strips were connected to the bottom-side ground plane with a 6-mil diameter conducting vias located at the centerline of the strips and spaced every 50 mils. To simulate the appropriate CPW mode propagation, three series delta-gap sources are put at the ends of the outer ground strips with amplitude -1 and the center signal conductor with amplitude 1, respectively. That is, a source is connected at the input port (center line), while two out-ofphase sources are excited from top and bottom strips at the same time. The circular vias, small compared to the guided wavelength, are approximated by rectangular vias constructed by two triangular cells because of the simpler discretization. Fig. 10 shows the transmission coefficient for an abrupt

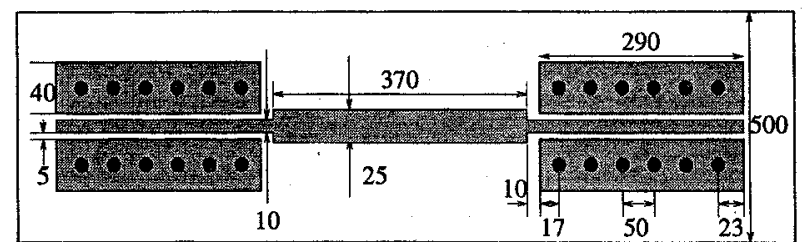

unit: 0.001 inch, via diameter: 0.006 inch

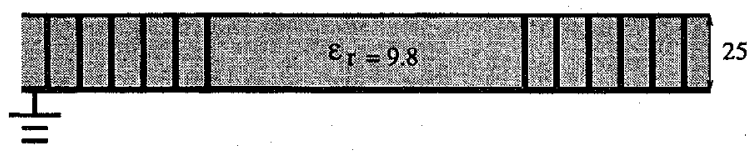

Fig. 9. GCPW circuit with microstrip-abrupt transition.

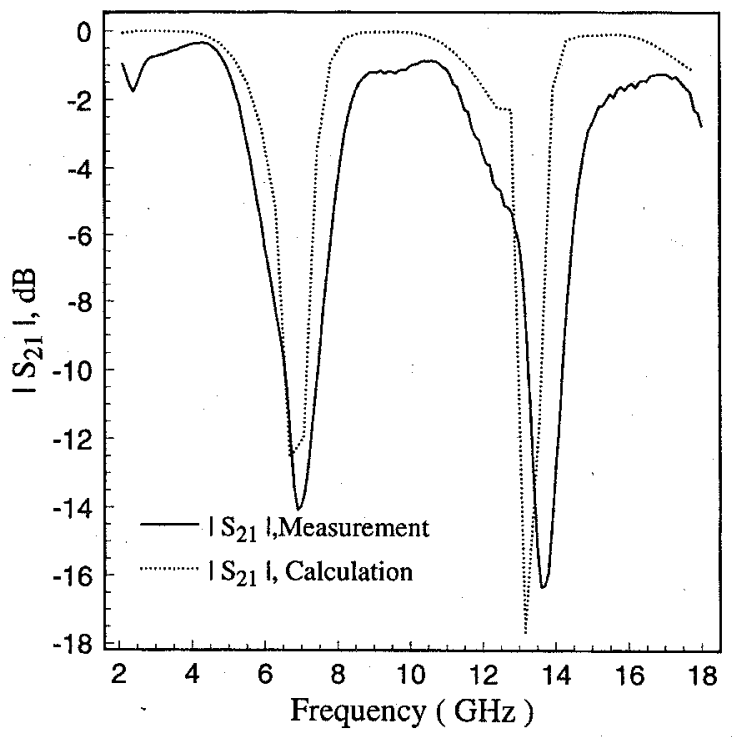

Fig. 10. Comparison of the transmission coefficient for Fig. 9 without vias.

transition without vias. Substantial radiation from the "microstrip" center conductor is expected since the underside ground plane is no longer connected to the CPW return strips. Two dips of $S_{21}$ at $6.9 \mathrm{GHz}$ and $13.6 \mathrm{GHz}$ are predicted very well. Fig. 11 shows the amplitude of the reflection and transmission coefficients for the GCPW circuit. It can be seen that the via effects help the broadband transmission of the signals from port 1 to port 2 . The calculated data shows good agreement with the measured data at higher frequencies, except for the shift in resonant frequency. The lower resonance at about $4 \mathrm{GHz}$ is missed in the calculation. The reason for this error is that this analysis assumes infinite ground planes and does not take into account the finite half-inch-width fabricated circuits. The assumption of infinite substrates is only an approximation for comparison with the fabrication circuits, especially at lower frequencies. There is significant radiation loss from the finite board. This loss can be verified from the sum of reflected and transmitted power as demonstrated in Fig. 12.

Another GCPW circuit is shown in Fig. 13. This circuit has similar dimensions and via parameters as the previous example, except this circuit has right-angle-bend transitions in place of a step discontinuity. The simulation results and measured data are shown in Fig. 14. Because of the very low reflection, the associated numerical errors make it difficult 


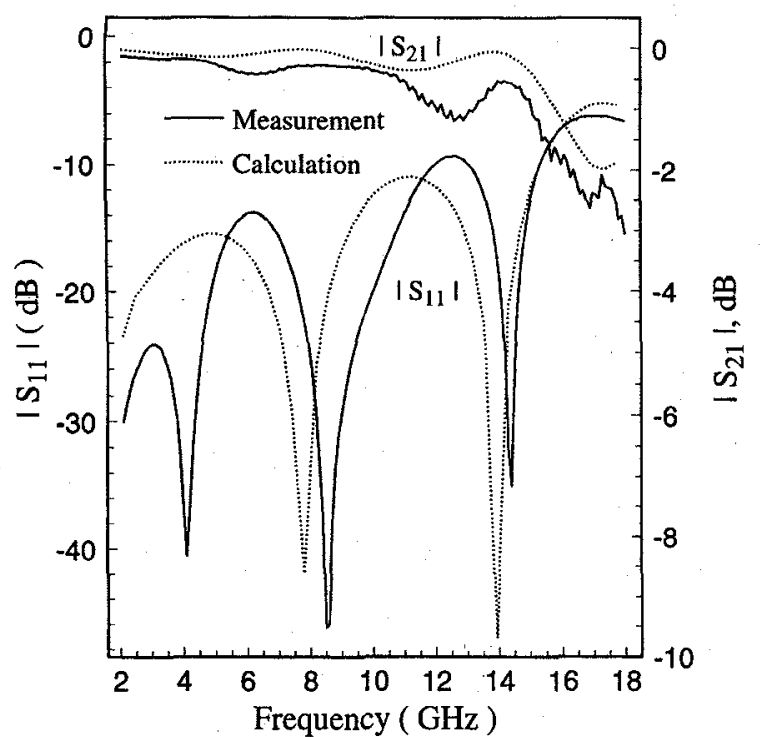

Fig. 11. Comparison of reflection and transmission coefficients for Fig. 9.

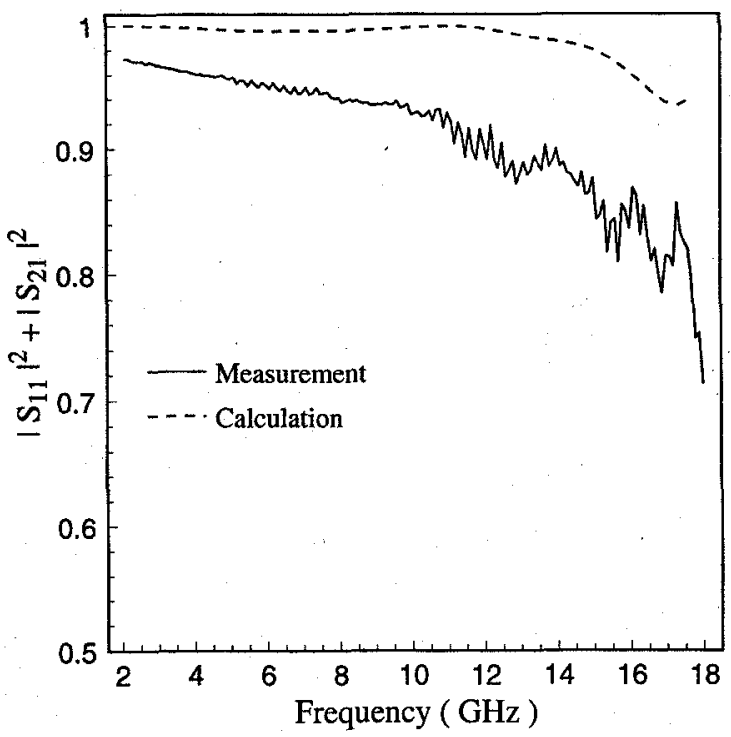

Fig. 12. Comparison of the sum of reflected and transmitted power for Fig. 9.

to simulate accurately: The resonant behavior between the measured and predicted results agree well. In addition, two dips of $\left|S_{21}\right|$ shown near $16 \mathrm{GHz}$ are predicted by this analysis. Again, there is severe radiation loss from the edges of the board as shown in Fig 15 . Nevertheless, this analysis yields pretty good simulation results for both cases in the frequency range of interest, 7-15 GHz. Commercial software based upon the finite-element method (FEM) suffers from very slow convergence because it treats the designed CPW modes of the GCPW circuits as higher order modes. Moreover, either FEM or FDTD analysis needs to grid the entire volume, including the open space in the air region, so huge computer resources, such as parallel computing processors and large memory usage, are necessary. This moment-method algorithm meshes only conductors, and therefore, a more efficient analysis can be achieved.

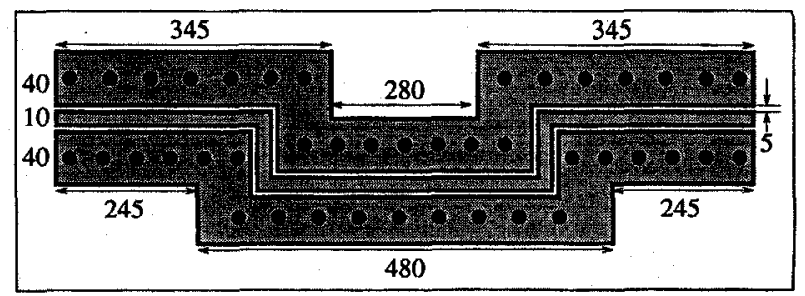

unit: 0.001 inch, via diameter: 0.006 inch, 0.05 inch apart for each via

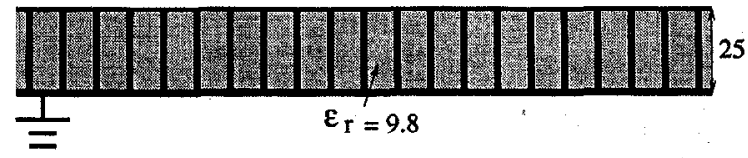

Fig. 13. GCPW circuit with right-angle-bend transition.

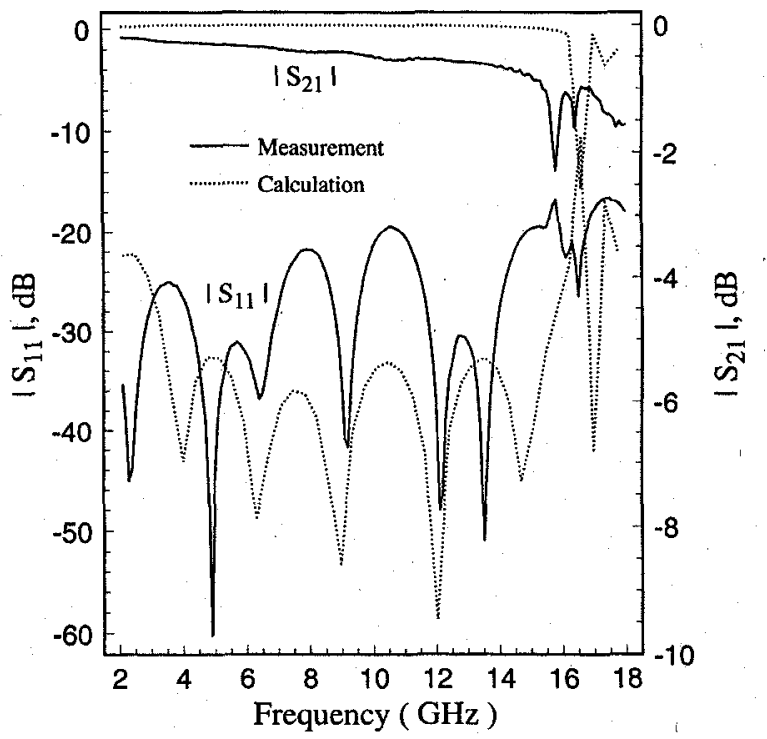

Fig. 14. Comparison of reflection and transmission coefficients for Fig. 13.

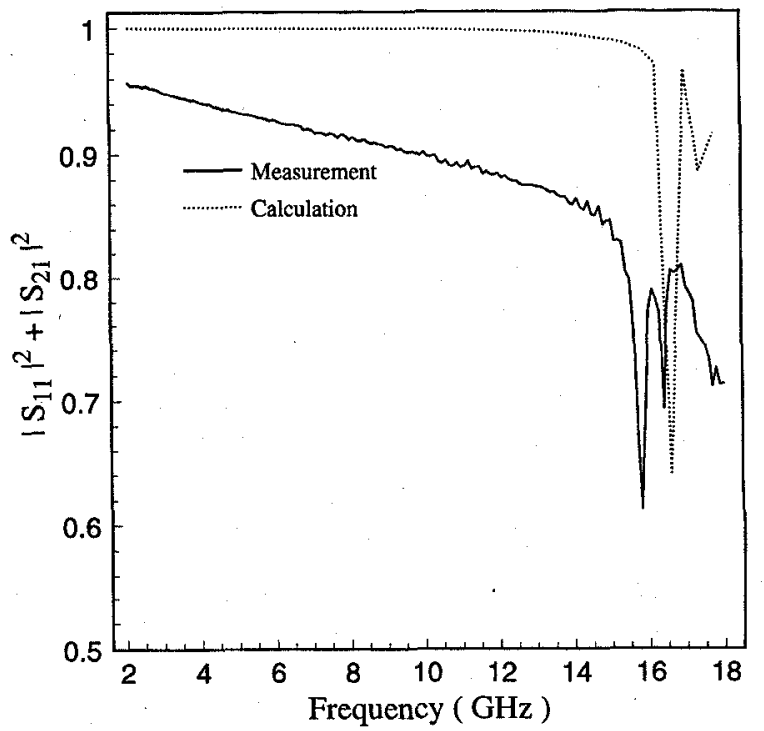

Fig. 15. Comparison of the sum of reflected and transmitted power for Fig. 13. 


\section{CONCLUSION}

In this analysis, the combined MPIE-EFIE technique is developed to model 3-D printed circuits of arbitrary shape in a multilayered medium. With the triangular patch function and vertical volume current basis with a triangular cross section, arbitrarily shaped microstrips and via holes can be analyzed accurately and efficiently. All circuit parameters, including the radiation and surface-wave characteristics, can be investigated from the current distribution which resulted from a moment-method analysis. Several examples with viahole and air-bridge transitions in multilayered structures were presented. Good agreement was found between experiments and published results. The presented analysis may find many applications in MMIC-CAD designs.

\section{APPENDIX}

The dyadic Green's function used in (7)-(9) has been formulated for multilayered media in [16]. To show the special treatment of analytic integration over $\hat{z}$ axis for the evaluation of matrix elements due to vertical current basis, the Green's function is reduced, for simplicity, to the expressions for a single-layered substrate. They are listed as follows:

$$
\begin{aligned}
& G_{A}^{x x}(\rho)=G_{A}^{y y}(\rho) \\
& =\frac{1}{2 \pi} \int_{0}^{\infty}\left[\frac{\mu_{0} \mu_{r} \tanh \left(\gamma_{1} d\right)}{D_{e}} J_{0}\left(k_{\rho} \rho\right) k_{\rho}\right] d k_{\rho} \\
& G_{A}^{v h^{\prime}}(\rho)=\int_{0}^{d} G_{A}^{v h}(\rho, z) d z \\
& =\frac{1}{2 \pi} \int_{0}^{\infty}\left[\frac{\mu_{0} \mu_{r}\left(\mu_{r} \epsilon_{r}-1\right) \tanh ^{2}\left(\gamma_{1} d\right)}{\gamma_{1} D_{e} D_{m}}\right. \\
& \left.\cdot J_{0}\left(k_{\rho} \rho\right) k_{\rho}\right] d k_{\rho} \\
& G_{q}(\rho)=\frac{1}{2 \pi} \int_{0}^{\infty}\left[\frac{D_{e}^{\prime} \tanh \left(\gamma_{1} d\right)}{\epsilon_{0} D_{e} D_{m}} J_{0}\left(k_{\rho} \rho\right) k_{\rho}\right] d k_{\rho} \\
& G_{E}^{z z}\left(\rho, z, z_{s}\right)=\frac{1}{2 \pi} \int_{0}^{\infty} \tilde{G}_{E}^{z z}\left(k_{\rho}, z, z_{s}\right) J_{0}\left(k_{\rho} \rho\right) k_{\rho} d k_{\rho} \\
& \tilde{G}_{E}^{z z}\left(k_{\rho}, z, z_{s}\right)=-\frac{1}{j \omega \epsilon_{0} \epsilon_{r}} \delta\left(z-z_{s}\right) \\
& +\frac{k_{\rho}^{2} \cosh \left(\gamma_{1} z_{s}\right)}{j \omega \gamma_{1} \epsilon_{0} \epsilon_{r} \cosh \left(\gamma_{1} d\right) D_{m}} \\
& \cdot\left\{\gamma_{0} \epsilon_{r} \sinh \left[\gamma_{1}(d-z)\right]+\gamma_{1}\right. \\
& \left.\cdot \cosh \left[\gamma_{1}(d-z)\right]\right\} \\
& G_{E}^{z z^{\prime}}(\rho)=\int_{0}^{d} \int_{0}^{d} G_{E}^{z z}\left(\rho, z, z_{s}\right) d z d z_{s} \\
& =\frac{1}{2 \pi j \omega \epsilon_{0}} \int_{0}^{\infty}\left[\left(\frac{d k_{0}^{2} \mu_{r}}{\gamma_{1}^{2}}-\frac{k_{\rho}^{2} \gamma_{0} \tanh \left(\gamma_{1} d\right)}{\gamma_{1}^{3} D_{m}}\right)\right. \\
& \left.\cdot J_{0}\left(k_{\rho} \rho\right) k_{\rho}\right] d k_{\rho}
\end{aligned}
$$

with the following parameters:

$$
\begin{aligned}
\gamma_{0} & =\sqrt{k_{\rho}^{2}-k_{0}^{2}} \\
\gamma_{1} & =\sqrt{k_{\rho}^{2}-k_{0}^{2} \mu_{r} \epsilon_{r}} \\
D_{e} & =\gamma_{0} \mu_{r} \tanh \left(\gamma_{1} d\right)+\gamma_{1} \\
D_{m} & =\gamma_{1} \tanh \left(\gamma_{1} d\right)+\gamma_{0} \epsilon_{r} \\
D_{e}^{\prime} & =\gamma_{1} \mu_{r} \tanh \left(\gamma_{1} d\right)+\gamma_{0} \\
\rho & =\sqrt{\left(x-x_{s}\right)^{2}+\left(y-y_{s}\right)^{2}}
\end{aligned}
$$

where $\rho$ is the radial distance, and $k_{\rho}$ is the spectral variable.

\section{ACKNOWLEDGMENT}

The authors extend their appreciation to Hughes Aircraft Company for the use of measured data collected by $\mathrm{H}$. Byrd of the Hughes Solid-State Microwave Laboratory for the GCPW circuits and to Dr. R. T. Kihm and Dr. K. Virga for many helpful insights and technical discussions.

\section{REFERENCES}

[1] T. Y. Wang, R. F. Harrington, and J. R. Mautz, "The equivalent circuit of a via," Trans. Soc. Comput. Simulation, vol. 4, pp. 97-123, Apr. 1987.

[2] _ , "Quasistatic analysis of a microstrip via through a hole in a ground plane," IEEE Trans. Microwave Theory Tech., vol. 36, no. 6, pp. 1008-1013, June 1988.

[3] A. Christ and H. L. Hartnagel, "Three-dimensional finite-difference method for the analysis of microwave-device embedding," IEEE Trans. Microwave Theory Tech., vol. MTT-35, no 8, pp. 688-696, Aug. 1987.

[4] A. Maeda, T. Kashıwa, and I. Fukai, "Full wave analysis of propagation characterstics of a through hole using the finite-difference time-domain method," IEEE Trans. Microwave Theory Tech., vol. 39, no. 12, pp. 2154-2159, Dec. 1991.

[5] W. J. R. Hoefer, "The transmission-line matrix method-theory and applications." IEEE Trans. Microwave Theory Tech., vol. MTT-33. no. 10. pp. 882-893, Oct. 1985.

[6] R. Sorrentino, F. Alessandrı, M. Mongiardo, G. Avitabrle, and L. Roselli, "Full-wave modeling of via hole grounds in microstrip by 3-D mode matching technique," IEEE Trans. Microwave Theory Tech., vol. 40, no. 12, pp. 2228-2234. Dec. 1992.

[7] T. Becks and I. Wolff, "Analysis of 3-D metallization structures by a full-wave spectral domain technique," IEEE Trans. Microwave Theory Tech. vol. 40, no. 12. pp. 2219-2227, Dec. 1992.

[8] M.-J. Tsai, T -S. Horng and N. G. Alexopoulos, "Via holes, bond wire and shorting pin modeling for multi-layered circuits," in IEEE MTT-S Int. Microwave Symp., San Diego, CA, 1994, vol. 3, pp. 1777-1780.

[9] S.-G. Hsu and R.-B. Wu. "Full wave characterization of a through hole via using the matrix-penciled moment method," IEEE Trans. Microwave Theory Tech., vol. 42, no. 8, pp. 1540-1547, Aug. 1994.

[10] K. A. Michalski, "The mixed-potential electric field integral equation for objects in layered media," Arch. Elek. Übertragung., vol. 39, pp. 317-322, Sept.-Oct. 1985.

[11] J. R. Mosig and F. E. Gardiol, "General integral equation formulation for microstrip antennas and scatterers," Proc. Inst. Elect. Eng., vol. 132, pt. H, no. 7, pp. 424-432, Dec. 1985.

[12] J. R. Mosig, "Arbitrarily shaped microstrip structures and their analysis with a mixed potential integral equation," IEEE Trans. Microwave Theory Tech., vol. 36, no. 2, pp. 314-323, Feb. 1988.

[13] S. M. Rao, D. R. W1lton, and A. W. Glisson, "Electromagnetic scattering by surfaces of arbitrary shape," IEEE Trans. Antennas Propagat., vol. AP-30, pp. 409-418, May 1982.

[14] M.-J. Tsai and N. G. Alexopoulos, "Proximity coupled microstrip elements and interconnects of arbitrary shape in multilayered medra." in IEEE MTT-S Int. Microwave Symp., Orlando, FL, 1995, vol. 2, pp. $475-478$.

[15] _ "Electromagnetically coupled microstrip ring-type antennas of arbitrary shape," in IEEE AP-S Int. Symp., Newport Beach, CA, 1995, vol. 1, pp. 684-687.

[16] M.-J. Tsal, C. Chen, and N. G. Alexopoulos, "Multi-layered structure Green's function for microstrip elements of arbitrary shape," in Proc. Int. 
Conf. Electromagnetics in Advanced Applications (ICEAA 95), Torino, Italy, 1995, pp. 3-5.

[17] W. E. McKinzie, "Electromagnetic modeling of conductor-backed aperture antennas and circuits of arbitrary shapes," Ph.D. dissertation, Univ. California, Los Angeles, 1992.

[18] D. R. Wilton, S. M. Rao, A. W. Glisson, D. H. Schaubert, O. M. AlBundak, and C. M. Butler, "Potential integrals for uniform and linear source distribution on polygonal and polyhedral domains," IEEE Trans. Antennas Propagat., vol. AP-32, no. 3, pp. 276-281, Mar. 1984.

[19] M. Abramowitz and I. A. Stegun, Eds., Handbook of Mathematical Functions with Formulas, Graphs, and Mathematical Tables, 10th ed., Dover, NY: 1972.

[20] K. L. Finch and N. G. Alexopoulos, "Shunt posts in microstrip transmission lines," IEEE Trans. Microwave Theory Tech., vol. 38, no. 11, pp. 1585-1594, Nov. 1990.

[21] "MIC Simulation Column," Int. J. Microw. Millimeter-Wave Comput. Aided Eng, vol. 3, no. 1, p. 76, 1993.

Ming-Ju Tsai (''93-M'96), for a photograph and biography, see this issue, p. 2465 .

Chinglung Chen (S'92-M'96), for a photograph and biography, see this issue, p. 2464.
Nicolaos G. Alexopoulos (F'86), for a photograph and biography, see this issue, p. 2465.

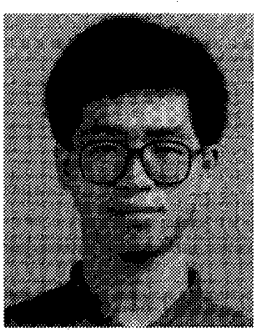

Tzyy-Sheng Horng (S'88-M'92) was born in Taichung, Taiwan, R.O.C., on December 7, 1963 $\mathrm{He}$ received the B.S.E.E. degree from National Taiwan University in 1985 and the M.S.E.E. and $\mathrm{Ph} . \mathrm{D}$. degrees from the University of California, Los Angeles, in 1990 and 1992, respectively.

From 1985 to 1987, he served in the R.O.C. Air Force as an Anti-Aircraft Artillery Officer. Since 1992, he has been an Associate Professor of the Electrical Engineering Department, National Sun Yat-Sen University, Kaohsiung, Taiwan. His current research interest is in the area of electromagnetic simulations of microwave integrated circuits.

Dr. Horng won the URSI Young Scientist Award in 1996. He is a Member of the Electromagnetics editorial board and a Committee Member of the Microwave Society of R.O.C. 